\title{
Quelques aspects du chant polyphonique traditionnel en Calabre
}

Some aspects of traditional vocal polyphony in Calabria

\section{Antonello Ricci}

\section{(2) OpenEdition}

\section{Journals}

Édition électronique

URL : http://journals.openedition.org/ethnomusicologie/1402

ISSN : 2235-7688

Éditeur

ADEM - Ateliers d'ethnomusicologie

Édition imprimée

Date de publication : 31 octobre 1993

Pagination : 87-98

ISBN : 2-8257-0485-7

ISSN : $1662-372 \mathrm{X}$

\section{Référence électronique}

Antonello Ricci, «Quelques aspects du chant polyphonique traditionnel en Calabre », Cahiers

d'ethnomusicologie [En ligne], 6 | 1993, mis en ligne le 02 janvier 2012, consulté le 20 avril 2019. URL : http://journals.openedition.org/ethnomusicologie/1402

Ce document a été généré automatiquement le 20 avril 2019.

Tous droits réservés 


\title{
Quelques aspects du chant polyphonique traditionnel en Calabre
}

Some aspects of traditional vocal polyphony in Calabria

\author{
Antonello Ricci
}

1 Ce texte présente certains aspects de la polyphonie chantée de tradition orale ${ }^{1}$ tels qu'ils sont apparus au cours d'une longue recherche de terrain que Roberta Tucci et moi-même avons fait, à partir du milieu des années 1970 et que nous poursuivons encore, sur la musique traditionnelle de la Calabre ${ }^{2}$.

2 La Calabre est la région la plus méridionale de l'Italie. Elle est baignée sur trois côtés par la mer et a un territoire essentiellement montagneux, aux rares zones plates. L'économie de la région se base surtout sur l'agriculture, sur l'élevage des moutons et sur la pêche ; récemment, elle a aussi reçu une forte impulsion dans le secteur touristique, qui ne concerne presque exclusivement que ses côtes. Une bonne partie de sa population est encore imprégnée d'une culture archaïque de type agro-pastoral, transmise oralement et en partie liée à la vie communautaire. Dans un tel cadre, la musique occupe une place importante, et de nombreux événements, cérémoniels, rituels ou quotidiens, sont scandés par les sons et les chants de la tradition.

Dans ce cadre sommairement esquissé, la polyphonie, tant vocale qu'instrumentale, occupe une place de premier plan, aussi bien par la variété des formes existantes, que par l'importance, qui est reconnue socialement, de «faire de la musique ensemble». Les formations de chant polyphonique, les compagnie di suoni - pour employer un terme forgé par Carpitella - expriment d'une manière exemplaire la conception esthétique d'une culture musicale fondée sur la combinaison et la superposition continue des sons. A ce propos, il est caractéristique que certains instruments musicaux parmi les plus archaïques soient polyphoniques (la cornemuse, la flûte double) et, dans le chant, on apprécie aussi beaucoup l'effet sonore engendré par un ensemble de plusieurs voix. 
4 La qualité de la voix est le premier élément qui apparaît à l'écoute et qui, en substance, contribue à créer un point commun entre des répertoires musicaux différents. L'émission vocale est déterminée par des techniques qui ne sont pas codifiées en règles et en manières d'apprendre, dérivant d'un modèle apprécié et partagé par la communauté sur la base d'un goût musical commun. La sonorité particulière de la voix semble découler d'une formulation qui prévoit l'emploi du premier registre vocal même dans les textures les plus aiguës ; l'émission "à gorge serrée ", comme si les muscles de la gorge tendent à se contracter et à se bloquer lorsque la mélodie monte vers l'aigu ; l'ouverture limitée de la bouche qui a pour conséquence de solliciter certains points de résonance des cavités nasale et buccale.

Combinées entre elles, ces composantes produisent une sensation accentuée de tension de la voix, un timbre tantôt guttural tantôt nasal, que l'on retrouve aussi bien dans le chant masculin que dans le chant féminin. Ces caractéristiques vocales ne sont pas exclusives à la Calabre, mais elles appartiennent à toute l'aire culturelle centrale et méridionale italienne et elles ont aussi été mises en évidence par d'autres musicologues et musiciens, dont Roberto De Simone (1979), Serena Facci (1985), Roberto Leydi (1973), Alan Lomax (1956, 1968), Giovanna Marini (1982).

6 Certains effets sonores qui caractérisent la voix sont souvent employés. Ce sont des coups de glotte de différentes intensités, des hoquets et des sanglots qui accentuent certains passages du chant, des aspirations qui dérivent de la prononciation dialectale. La position du corps pendant le chant semble elle aussi liée à l'émission sonore. En tenant son buste légèrement plié en avant, le chanteur imprime à la voix une poussée en avant. Souvent, il met sa main à côté de sa bouche (cf. fig. 1) pour mieux diriger le chant ou corriger l'intonation de la voix. Dans les groupes de polyphonie vocale, la disposition des personnes est, elle aussi, fonction de la bonne réussite du chant. Souvent, elles se disposent en cercle, pour mieux parvenir à la fusion parfaite des voix (cf. fig. 2), dite, en dialecte accùordu (accord) quand, comme le disent les chanteurs, on n'entend qu'une seule voix. Dans les chants accompagnés par des instruments de musique, les chanteurs sont toujours tournés vers l'instrument pour « en prendre » les sons et pour diriger leurs propres voix vers lui (cf. fig. 3).

7 Comme nous l'avons dit, la polyphonie est très répandue en Calabre, sous des formes exclusivement vocales, comme les chants dits all'aria (à l'air) recueillis dans la province de Cosenza, ou comme certains répertoires des minorités linguistiques calabro-albanaises, aussi bien qu'avec accompagnement d'instruments musicaux. 
Fig. 1 : Groupe polyvocal de Sartano (Carolina Logullo, Concetta Mollo, Ida Cariati et Elvira Trombini). Position a schiera. Noter la main à coté de la bouche

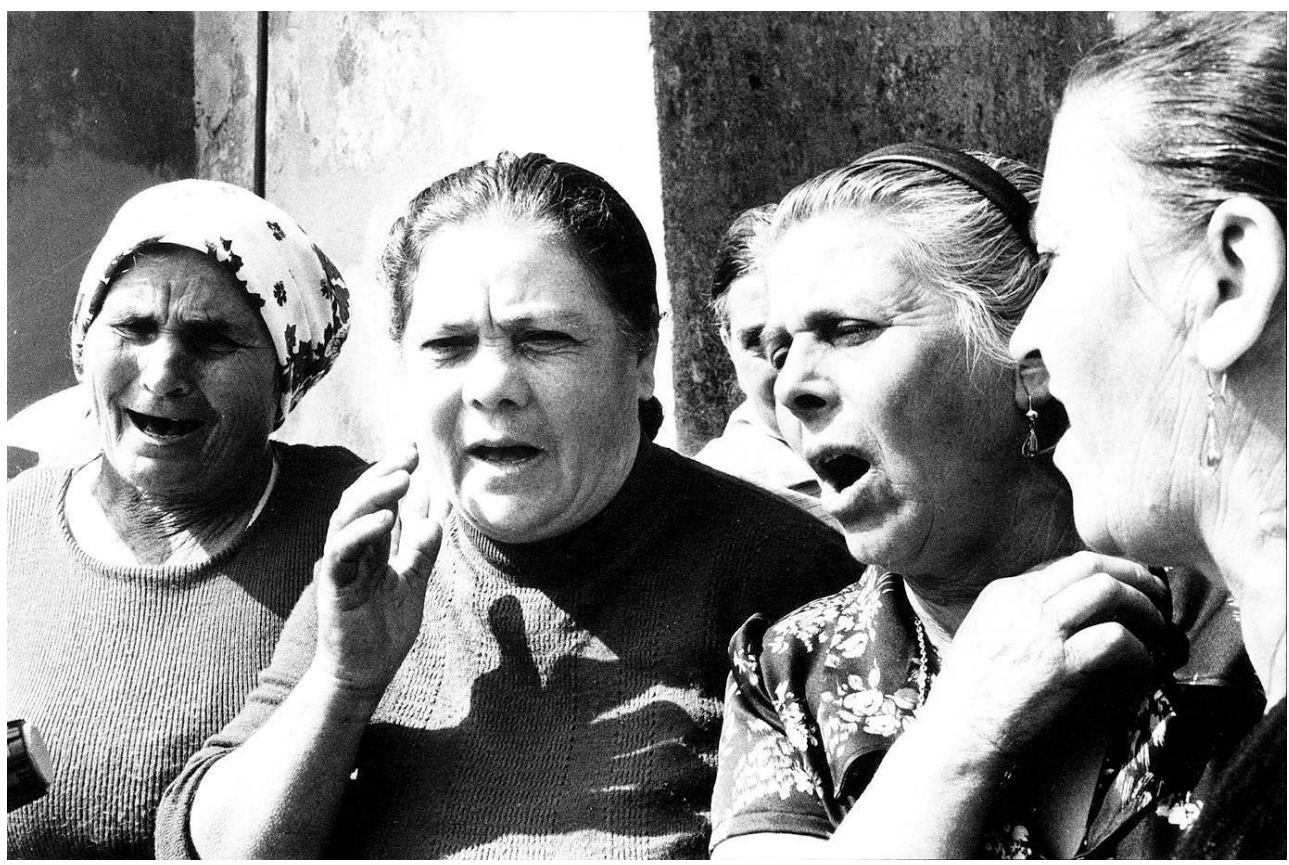

Photo : A. Ricci, 1981

Fig. 2 : Groupe polyvocal de Torano Castello (Mafalda Sangineto, Emilia Perrone, Rosaria Mazzei et Iolanda Docimo). Position a cerchio

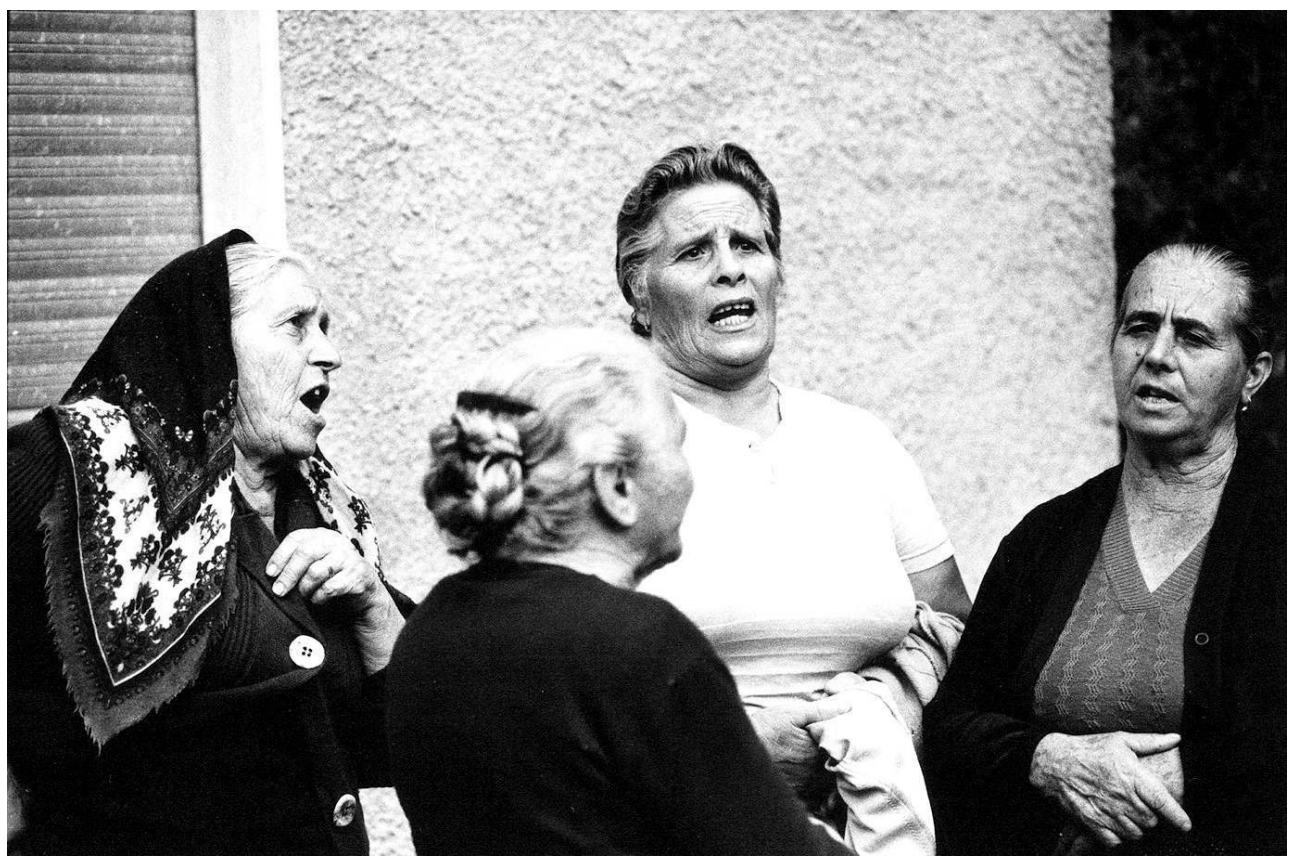

Photo : A. Ricci. 1984

8 Commençons par examiner un morceau accompagné. Il s'agit d'un chant qui fait partie du répertoire de la chitarra battente ${ }^{3}$ (un instrument de musique encore très fréquent dans la musique traditionnelle calabraise) recueilli à Mesoraca, dans la province de Catanzaro. Il s'appelle Cioparedda, à cause du mot que l'on rajoute à la fin de chaque strophe chantée. 
9 Dans l'exemple que nous allons prendre, la mélodie principale, celle sur laquelle s'articule aussi le texte verbal, est exécutée par le chanteur soliste. Comme dans de nombreuses autres formes de chant de la tradition populaire calabraise, les vers sont manipulés : il sont tronqués, on y fait des adjonctions et on les recompose, créant ainsi des formes de versification et des strophes parfois très élaborées. Dans ce cas, le texte verbal se base sur une séquence de distiques d'hendécasyllabes à rime plate. Chaque strophe chantée a pour origine un unique vers au début duquel on ajoute des syllabes ou des voyelles afin d'obtenir une attaque en battre. La structure de la strophe chantée prévoit une première partie composée par le vers en entier auquel est ajoutée l'expression oi bella; une seconde partie comprend la seconde moitié du vers plus le mot cioparedda :

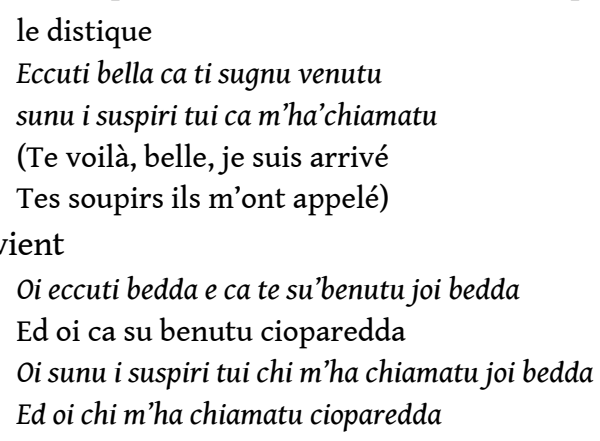

10 A chacune de ces deux parties correspond une phrase mélodique. Les deux autres voix d'accompagnement entrent dans la deuxième moitié des deux phrases mélodiques. Elles exécutent principalement un chant avec des syllabes dépourvues de signification. Dans la première intervention sont exécutées deux notes longues (respectivement le troisième et le deuxième degré de l'échelle) en manière de bourdon ; dans la seconde intervention, qui caractérise fortement l'expressivité de la dernière partie de la strophe, la mélodie principale est exécutée à l'unisson avec les voix d'accompagnement. Toujours du point de vue expressif, on remarquera la dynamique très rude de l'interprétation: les finales contribuent, elles aussi, à définir le style, en se terminant par une dernière note ayant une appoggiature particulière. 
Fig. 3 : Chant accompagné par la chitarra battente (Basile e Salvatore Cariati)

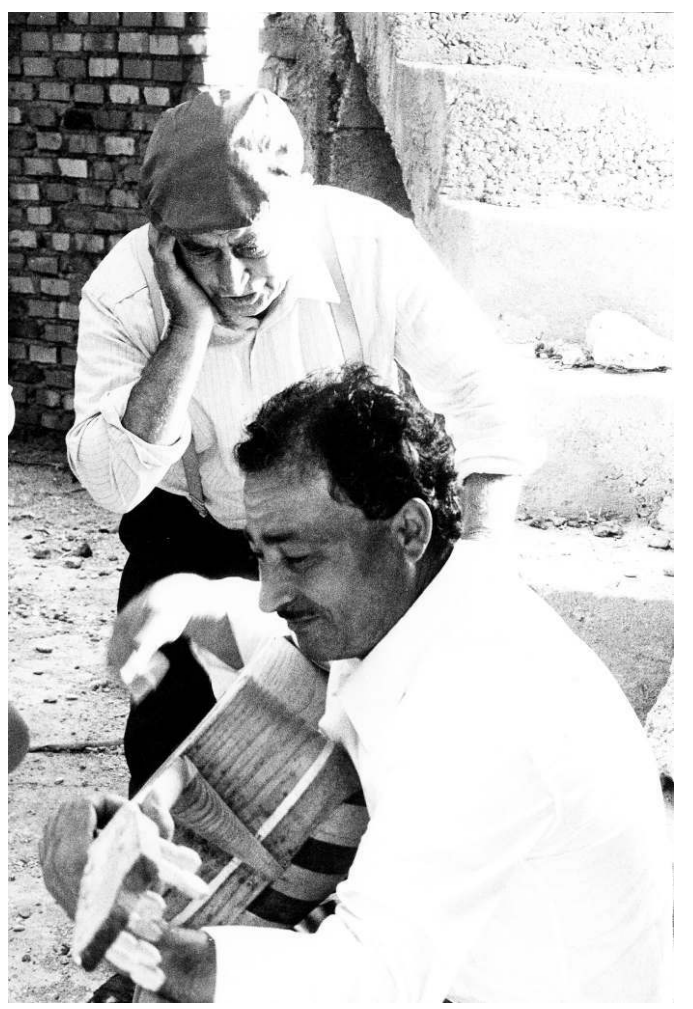

Noter la position du chanteur tourné vers l'instrument et sa main à coté de la bouche Photo : A. Ricci, 1981

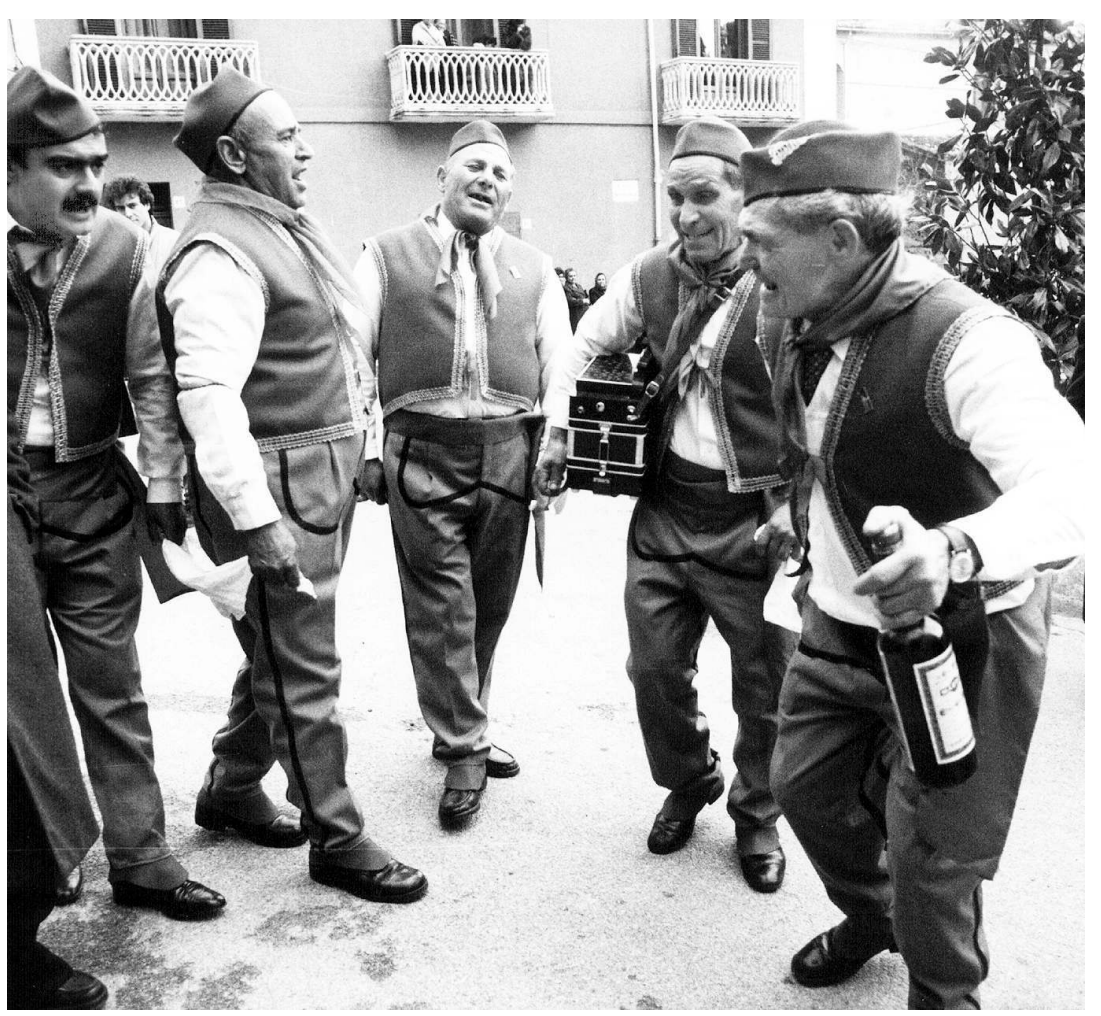



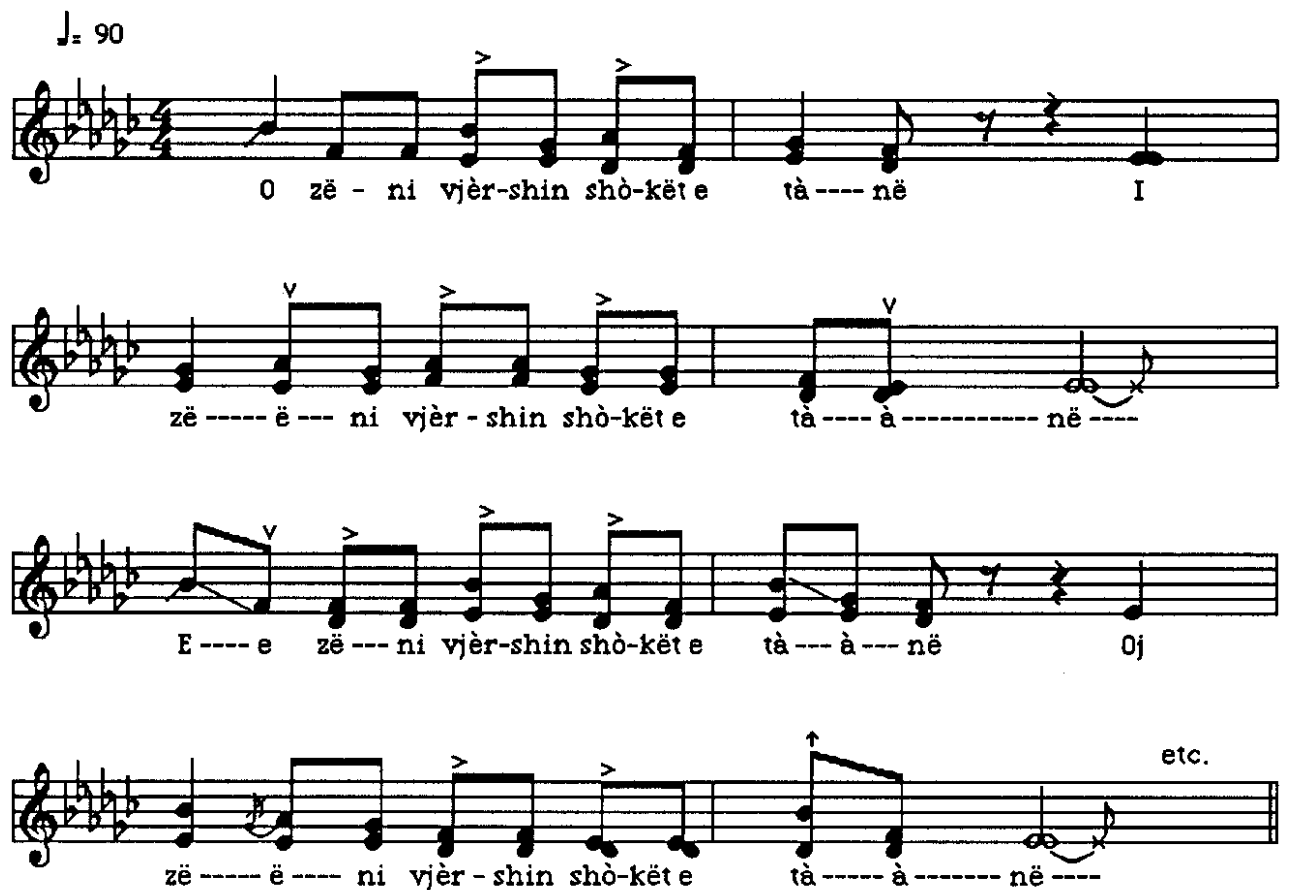

(A. Ricci)

11 Deux formes de chant plurivocal en langue albanaise ${ }^{4}$ ont été recueillies à Civita, dans la province de Cosenza, au cours de la Valia, la fête que cette communauté célèbre chaque année, après Pâques. Le premier morceau (cf. exemple musical $n^{\circ} 1$ ) est un chant rituel, dénommé viershë (vers), exécuté par deux groupes de cinq hommes au cours d'une quête itinérante. Les dix chanteurs, se tenant unis entre eux par des mouchoirs, parcourent en file les rues du village, en chantant des vœux. En échange, ils recoivent à boire et à manger (cf. fig. 4). Les viershë ont une structure responsoriale : chaque strophe, composée d'un vers répété deux fois, est exécuté par deux sous-groupes de cinq chanteurs, qui se répondent en alternant. Chaque sous-groupe est dirigé par une voix soliste. La polyphonie est à deux parties : la voix soliste entonne le chant auquel s'ajoutent les quatre voix qui restent, à l'unisson entre elles, qui la suivent selon une ligne pratiquement parallèle, en général une tierce en-dessous, mais parfois aussi à des intervalles de quinte. La polyphonie vocale à deux parties, elle aussi, n'est pas une exclusivité de la Calabre, mais elle est répandue, sous différentes formes, dans toute l'Italie ${ }^{5}$. Il n'y a pas de pause d'une strophe à l'autre; chaque fois, le soliste attaque sur la « coda» du groupe précédent, ce qui produit une véritable chaîne de strophes. Dans ce morceau, on remarquera surtout le style âpre et agressif, comme une sorte de guet-apens sonore qui surprend l'auditeur, en particulier lorsque le chant est entonné.

Le deuxième morceau est une sérénade d'amour exécutée par trois chanteurs. L'enregistrement a été effectué un soir, au cours d'une réunion entre amis, dans la cave de l'un d'entre eux. Le texte verbal se base sur un vers répété deux fois. A chaque vers correspond une phrase mélodique. Le type de polyphonie est encore à deux parties avec une voix soliste qui fournit l'attaque du chant. Les voix d'accompagnement entrent immédiatement, quasiment avec le soliste. Le style de chant, là encore, est très âpre et agressif et il est caractérisé par de rapides glissandos de la voix soliste, soutenus par la 
base solide des autres voix qui, surtout dans la partie finale, accompagnent avec des bourdons.

13 Voyons maintenant quelques chants polyphoniques recueillis principalement dans deux villages: Torano Castello e Sartano ${ }^{6}$, province de Cosenza, où l'on a pu constater que l'habitude de chanter ensemble a bien résisté à l'usure du temps.

Exemple musical $\mathrm{N}^{\circ} 2$
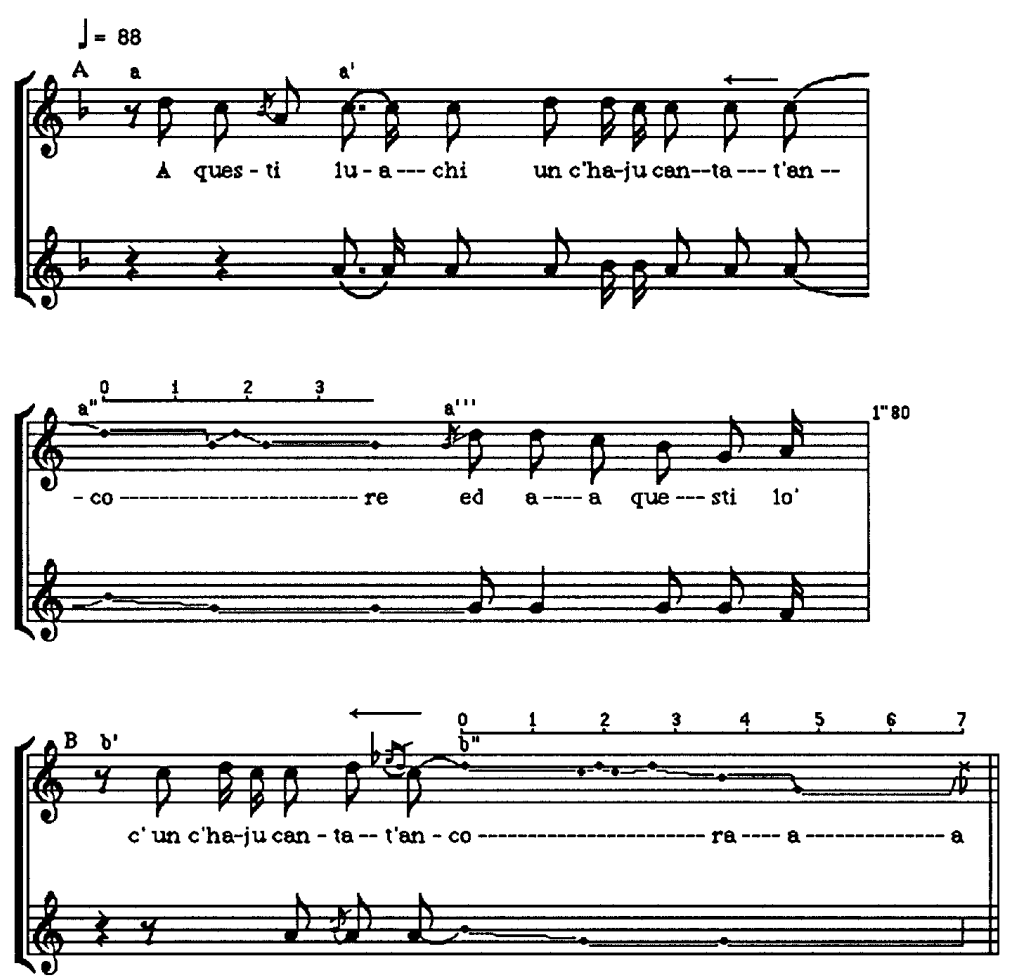

(A. Ricci) 


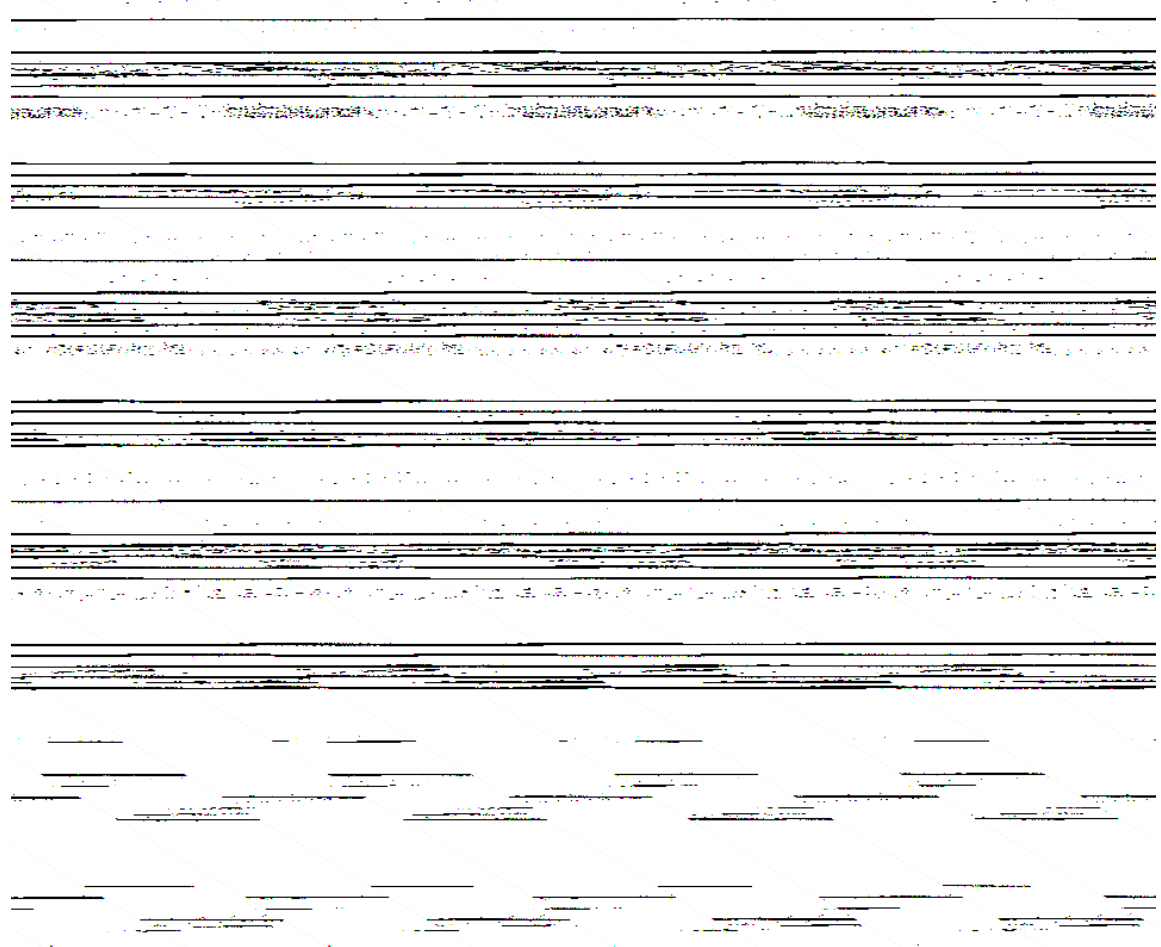

Le répertoire enregistré dans ces deux villages est en grande partie constitué de chants appelés all'aria. Ils représentent le noyau central du répertoire, aussi bien à cause de l'intérêt musical qu'ils suscitent que par leur nouveauté dans le cadre des recherches ethnomusicologiques italiennes, par rapport aux répertoires calabrais connus jusqu’à présent.

15 Les chants all'aria de Torano et de Sartano constituent un répertoire de polyphonie vocale quasi exclusivement féminin, qui était généralement exécuté durant les travaux agricoles. Il s'agit d'un répertoire spécialisé, qui prévoit des rôles musicaux bien définis : une voix soliste et des voix d'accompagnement. Les groupes féminins qui les exécutent peuvent être considérés comme ayant un niveau professionnel, si l'on tient compte de la considération dont ils jouissent. Souvent, par le passé, les chanteuses solistes étaient choisies pour travailler à la campagne justement en fonction de leurs qualités vocales.

Les chants all'aria, dans le lexique musical de ces deux villages, assument des dénominations particulières sur la base des différentes formes mélodiques (a voca regolare, $c f$. exemple musical $n^{\circ} 2$, a voca diritta, $c f$. exemple musical $n^{\circ} 3$, a bandieri bella, a voca 'e notte, etc.). Le terme voca que l'on retrouve fréquemment dans le lexique musical des chanteurs, possède une valeur sémantique très ample: en premier il signifie "voix ", puis, par extension, il indique aussi "chant », «mélodie ", «forme mélodique ». Cette pluralité de significations s'explique si l'on pense que, dans ce contexte, l'expérience musicale est vécue à travers l'emploi exclusif de la voix.

17 Les chants all'aria sont exécutés par des groupes composés généralement de trois ou quatre personnes, où la voix soliste (en dialecte chira chi canta avanti, « celle qui chante avant ») entonne le chant et les autres voix (en dialecte chire chi cantanu appriessu, « celles qui chantent après ») s'ajoutent après trois à cinq notes pour l'accompagner et la 
soutenir. Il s'agit d'une polyphonie en deux parties où l'accompagnement se réalise surtout par bourdons, mais parfois il y a alternance des parties à bourdon et des parties à parallèlisme. Dans un cadre mélodique restreint (de quinte ou de sixte), la capacité de la voix soliste d'improviser et de faire des mélismes donne souvent lieu à de véritables démonstrations de virtuosité vocale. Les notes ne sont presque jamais tempérées. Le dessin mélodique a généralement une tendance descendante. La cadence finale est surtout sur le final. Les voix d'accompagnement se meuvent dans un ambitus de tierce et de quarte, principalement entre elles, à l'unisson, ou bien deux ou plusieurs à l'unisson et une à l'octave supérieure. Dans ce dernier cas, la voix à l'octave supérieure est dite scùordu, selon une terminologie calabraise qui indique des sons perceptibles séparément des autres. La scansion rythmique, principalement binaire, n'est jamais définie d'une manière précise : souvent, après un début soutenu, les durées s'altèrent et se dilatent progressivement. Et, dans la finale de chaque strophe, l'exécution d'un cri bref et aigu dit: ' $u$ sguiddu, ' $u$ sgrillu est fréquente. Les textes verbaux des chants all'aria ont un caractère lyrique et ils traitent des thèmes les plus divers : l'amour, l'indignation, l'amitié, etc. D'un point de vue littéraire formalisé, ils se basent sur une séquence de distiques d'hendécasyllabes à rimes croisées. Dans l'exécution, ce modèle, qui est exprimé sous forme récitée par les chanteuses, subit un certain nombre de modifications - troncature de vers et de paroles, recompositions, rajout de paroles et de différents stéréotypes - qui donnent lieu à la formulation de nouvelles strophes. Par exemple, dans les chants a voca regolare, le distique :

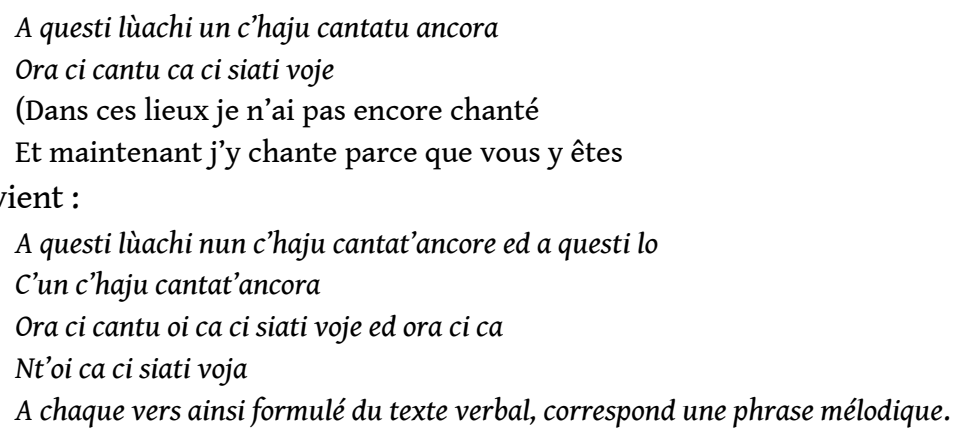

Le second exemple est une pièce enregistrée à Sartano et dénommée a voca diritta. Il s'agit d'un modèle mélodique très semblable au précédent. Naturellement, on peut y noter de nombreuses différences, surtout dans le style d'exécution qui est beaucoup plus âpre et plus riche en effets sonores que celui de Torano, dans la manière de tronquer les syllabes des paroles, dans les rajout de phrases stéréotypées. Dans ce cas, le même distique devient :

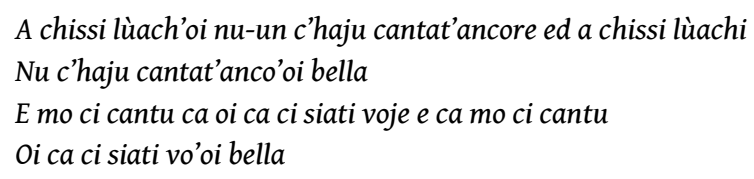

Cette manière d'intervenir sur le texte verbal est définie en dialecte canta'a stanzì ${ }^{7}$, chanter en procédant par troncatures et recompositions. Typique des chants all'aria est la dispenzata, la dédicace d'une chanson à une personne, à un lieu, etc. Elle consiste en un ou deux distiques à rime plate situés à la fin de la chanson.

Les chants all'aria étaient surtout exécutés à la campagne, durant les travaux agricoles les plus variés: la moisson, le vannage et la récolte des gerbes de blé, la moisson et le vannage des céréales, la cueillette des olives, le ramassage du bois dans les forêts, etc. Ce ne sont pas des chants de travail à proprement parler, qui aident rythmiquement le 
travail ; mais en accompagnant celui-ci, ils font passer le temps. Le travail fait en commun représente aussi le moment didactique le plus important pour apprendre les techniques polyphoniques. Quelques-unes des chanteuses que j'ai connues soutiennent qu'elles ont appris les chants lorsqu'elles étaient petites filles, de leurs camarades plus grandes et expertes, au cours des travaux effectués en leur compagnie.

\section{BIBLIOGRAPHIE}

AGAMENNONE Maurizio, Serena FACCI, 1982, « La trascrizione delle durate nella polivocalità popolare a due parti in Italia », Culture musicali I $1: 87-103$.

BIAGIOLA Sandro (éd.), 1986, Etnomusica. Catalogo della musica di tradizione orale nelle registrazioni dell'Archivio Etnico Linguistico-musicale della Discoteca di Stato. Roma : Discoteca di Stato-Il Ventaglio.

DE GAUDIO Innocenzo, 1990, Gli albanesi di Calabria. Canti bivocali delle comunità Arbëreshë (provincia di Cosenza). Opuscule annexé au disque de la collection Ricerche etnomusicologiche Archivio sonoro. Milano : Albatros VPA 8501.

DE SIMONE Roberto, 1979, Canti e tradizioni popolari in Campania. Roma : LatoSide.

FACCI Serena, 1985, «Quindici esempi di polifonia tradizionale », in : Forme e comportamenti della musica folklorica italiana. Edité par G. Giuriati. Milano : Unicopli : 45-93.

LEYDI Roberto, 1973, I canti popolari italiani. Milano : Mondadori.

LOMAX Alan, 1956, « Nuova ipotesi sul canto folkloristico italiano », Nuovi Argomenti 17-18 : 109-135.

LOMAX Alan, 1968, Folk Songs Style and Culture. Washington: American Association for the Advancement of Science.

MARINI Giovanna, 1982, « Dalla trascrizione di un canto contadino alla composizione di un pezzo per quartetto vocale », Musica/Realtà III $9:$ 107-122.

RICCI Antonello, 1991로 " « A chissi lochi un c'haju cantatu ancora. Canti polivocali della provincia di Cosenza », Quaderni Calabresi 73 : 42-54.

RICCI Antonello, 1991b, « Polivocalità tradizionale di Torano Castello e Sartano ». Studi Musicali XX 1:3-38.

RICCI Antonello, Roberta TUCCI, 1982, Calabria 2 strumenti la chitarra battente. Opuscule annexé au disque de la collection I Suoni. Milano : Fonit Cetra SU 5008.

RICCI Antonello, Roberta TUCCI, 1984, « Il canto “alla lonnuvucchisa”. Analisi del testo verbale », Culture musicali III 5-6 : 199-268.

TUCCI Roberta, Antonello RICCI, 1985, « The chitarra battente in Calabria », The Galpin Society Journal XXXVIII: 78-105. 


\section{NOTES}

1. La polyphonie chantée de tradition orale en Calabre est un sujet sur lequel j'ai depuis longtemps déjà orienté mon activité de recherche. Elle a été le thème d'un mémoire de licence en ethnomusicologie intitulé Canti polivocali calabresi: Torano Castello e Sartano que j'ai soutenu à l'Université «La Sapienza » de Rome, sous la direction de Diego Carpitella. A partir de ce travail ont été élaborés deux essais qui traitent les aspects sociaux et le contexte dans lesquels les chants sont exécutés (1991a) et les aspects plus nettement musicaux du répertoire polyvocal analysé (1991b). En outre, la plupart des morceaux dont il est question dans ce texte sont contenus dans le CD Folk Music of Calabria, Italy, « Traditional Music of the World 3 » BM 55803 (1991), édité par A. Ricci et R. Tucci, avec opuscule en annexe, publié par l'International Institute for Traditional Music de Berlin en collaboration avec l'International Council for Traditional Music.

2. On a surtout fait des enregistrements sonores et des photographies. Une partie des premiers se trouve à l'Archivio Etnico Linguistico-Musicale de la Discoteca di Stato de Rome, collections $146 \mathrm{M}, 163 \mathrm{M}$ et 166M, cf. Biagiola (1986). Une sélection de bandes magnétiques et de photographies se trouve au Centro per le Iniziative Musicali Siciliane de Palerme (matériel concernant les communautés calabro-albanaises) et au Centro Interdipartimentale di Documentazione Demoantropologica « Raffaele Lombardi Satriani » de l'Université de la Calabre. En outre, une collection de photographies se trouve à la Fototeca du Museo Nazionale delle Arti e Tradizioni Popolari, à Rome.

3. Sur la chitarra battente en Calabre, $c f$. Ricci-Tucci (1982) et Tucci-Ricci (1985).

4. Pour une recherche sur la polyphonie vocale calabro-albanaise (arbëreshë) réalisée dans différents villages de la Calabre, $c f$. De Gaudio (1990).

5. Sur cette question, $c f$. Agamennone-Facci (1982).

6. Sur les chants polyvocaux de Torano Castello et de Sartano, $c f$. Ricci (1991 a-b).

7. Canta'a stanzia a la même signification que l'expression cantare a strofette propre au lexique relatif aux chants accompagnés par la chitarra battente. Dans ces répertoires, les vers et les strophes sont organisés selon des règles métriques et musicales très rigoureuses. $C f$. Ricci-Tucci (1984).

\section{RÉSUMÉS}

In the small rural communities of Calabria, in southern Italy, polyphonic singing - which is purely vocal without any accompanying instrument, and practised by groups of women or men still fulfils important social and ritual functions. The author describes various circumstances calling for collective singing, whether in the context of work or during ceremonies and festivals. He also provides information on the way in which vocal ensembles are constituted, and the types of polyphonic singing which characterise the repertoires analysed. His data are based on field recordings undertaken in three Calabrese villages; the inhabitants of one of these speak Albanian. 


\section{AUTEUR}

\section{ANTONELLO RICCI}

Antonello Ricci est né en Calabre. Musicien et anthropologue, il a passé sa licence en ethnomusicologie avec Diego Carpitella et suit, actuellement, les cours du doctorat de recherche en ethnologie sous la direction de Luigi M. Lombardi Satriani, titulaire de l'enseignement d'Ethnologie I, Université de Rome «La Sapienza». Il mène depuis quinze ans des recherches de terrain sur les aspects les plus divers de la musique de tradition orale en Calabre. Actuellement il étudie l'immigration sénégalaise dans le Latium, et le pèlerinage pour la fête de saint Jacques à Santiago de Compostela. Il est l'auteur de nombreux articles et de deux disques d'ethnomusicologie (en collaboration avec Roberta Tucci) et l'auteur-interprète d'autres disques. 